\title{
ASSESSMENT OF CHANGES IN BLEEDING PATTERNS AFTER CHEMOTHERAPY IN WOMEN WITH BREAST CANCER USING CONTRACEPTIVES
}

Bruna Luisa Ferraço Lima, Luís Carlos Sakamoto, Sthephanie Avesani João Figueiredo Carneiro, Natália Kathllen Colli Afonso, Luiz Henrique Gebrim

${ }^{1}$ Hospital Pérola Byington, Centro de Referência da Saúde da Mulher - São Paulo (SP), Brazil.

Introduction: The evolution regarding chemotherapy treatment through new medications and new regimens in women with a history of breast cancer over time allowed better therapeutic responses to the disease. One of the consequences of chemotherapy concerns the impairment of the follicular reserve and its hormonal production. Objectives: To assess the importance of the bleeding pattern in women with a history of breast cancer who underwent chemotherapy and the impact on the follicular population in determining ovarian failure. Methods: Between August and December 2020, 419 women were seen at the Family Planning outpatient clinic of the Women's Health Reference Center (Hospital Pérola Byington), São Paulo, with 109 women having a history of breast cancer and undergoing a cross-sectional study, regarding the epidemiological characteristics of the care, the consequences to the bleeding pattern after chemotherapy treatment and the need for safe contraception. Results: The average age of women was 42.0 years, and the brown color was more prevalent in 49 women (45.0\%). The mean age at diagnosis of breast cancer was 37.9 years, with 104 women (95.4\%) presenting with invasive carcinoma and five cases (4.6\%) with carcinoma in situ. Among the types of invasive carcinoma, luminal type B was present in 42 women (40.4\%), HER2 in 25 (24.0\%), triple negative in 19 (18.3\%) and luminal A in 18 (17.3) \%). 91 women (83.5\%) underwent chemotherapy, and 82 other underwent hormonal treatment (75.2\%), with 78 of them (95.1\%) using tamoxifen. Obesity was present in 39 women (35.8\%). Regarding the menstrual pattern, 38 women (34.9\%) had amenorrhea after chemotherapy, but only six $(15.8 \%)$ had confirmed laboratory menopause. Contraception through the copper intrauterine device (IUD) was performed in 82 of them (75.2\%). Conclusions: Amenorrhea as a bleeding pattern after chemotherapy can be caused by impairment in the follicular population, causing ovarian failure and difficulty in pregnancy. On the other hand, contraception through a safe method such as copper IUDs until laboratory confirmation of menopausal status must be adopted. New studies should be carried out to better clarify this situation. 